\title{
ESCENARIOS CONTRÁFACTICOS1
}

\section{Counterfactual Scenarios}

\section{OSVAldo Pessoa JR.}

(Universidad de São Paulo, Brasil)

\begin{abstract}
Resumen
Definimos un "escenario contrafáctico", esto es, una situación posible que no se realizó, como una posibilidad futura en algún instante del pasado. El ejemplo más consagrado de análisis contrafáctico viene del área de la historia económica, donde se pueden hacer previsiones cuantitativas sobre el futuro. Esta posibilidad, aliada a la evaluación retrospectiva, dan un buen grado de confianza a este análisis. Otra área en la que se pueden postular escenarios contrafácticos es la historia de la ciencia. Lo que permite eso, no es la previsión del futuro sino, la ventaja retrospectiva de saber hoy de cuáles descubrimientos estaban próximos los científicos del pasado. Para eso, se presupone la objetividad de las ciencias naturales. Un tercer tipo de análisis contrafáctico reúne a la historia virtual, la evolución biológica y el desarrollo de juegos. Al final, utilizamos nuestra definición de escenario contrafáctico para explorar la cuestión de la identidad personal y los individuos transmundiales.
\end{abstract}

Palabras clave: Contrafácticos. Mundos posibles. Historia económica. Historia de la ciencia. Historia virtual. Identidad personal. Individuos transmundiales.

\begin{abstract}
A "countefactual scenario", i.e. a possible situation that did not happen, is defined as a future possibility in some instant of the past. The best example of counterfactual analysis is in the field of economic history, where one can make quantitative predictions about the future. This possibility, together with hindsight, give this analysis a good degree of reliability. Another area in which one may postulate counterfactual scenarios is the history of science. What allows this is not the prediction of future events, but the advantage of hindsight, of knowing today what discoveries the scientists of the past were close to making. This presupposes the objectivity of the natural sciences. A third kind of counterfactual analysis encompasses virtual histroy, biological evolution, and the progression of games. In the end, our definition of counterfactual scenario is used to explore the issue of personal identity and transworld individuals.
\end{abstract}

Keywords: Counterfactuals. Possible worlds. Economic history. History of science. Virtual history. Personal identity. Transworld individuals.

\footnotetext{
${ }^{1}$ Traducido del portugués al castellano por Lucas E. Misseri.
} 


\section{Escenarios contrafácticos}

Footfalls echo in the memory

Down the passage which we did not take

Towards the door we never opened

Into the rose-garden.

T.S. ELIOT (1935)

La historia podría haber sido diferente. Si la isla de Córcega hubiese sido vendida por la República de Génova a Francia apenas un año después de 1768 (cuando de hecho fue vendida), Napoleón Bonaparte no habría nacido con la ciudadanía francesa, y no se convertiría en general francés. Así, muy probablemente Portugal no habría sido amenazado por la invasión del Imperio Francés, y el rey Juan VI no habría venido al Brasil en 1808. Con eso, Río de Janeiro no habría conseguido la centralización de poder necesaria para evitar el desmembramiento de la colonia en varios países de lengua portuguesa, cuando ocurriesen los movimientos de independencia. ¡En lugar de un único país, la colonia portuguesa se tranformaría en varias naciones (como de hecho ocurrió en la América española), y hoy podríamos tener, además de Brasil (centrado en Río y en Minas), tal vez la República Río-Grandense, São Paulo, Bahía, la Confederación del Ecuador y la República del Gran Pará y Maranhão! (Cavalcante, 2002).

Esa especulación es un ejemplo de historia "virtual", como se dice en el área de historia (Ferguson, 1997; Roberts, 2004), o escenario “contrafáctico", como prefieren los filósofos analíticos. El pensamiento contrafáctico es un asunto también estudiado en psicología, y ocurre por ejemplo cuando nos arrepentimos de algo: "Si al menos hubiese escogido el camino más trillado!" If only... (Si al menos...) es un libro del psicólogo Neal Roese (2005) sobre el asunto, así como la selección What Might Have Been (Lo que podría haber acontecido) (Roese \& Olson, 1995). En Internet, hay también un sitio llamado Uchronia (término introducido por Renouvier, 1876), que hace una lista de novelas que imaginan escenarios contrafácticos para la historia.

\footnotetext{
1 "Ecoam passos na memória / Ao longo das galerias que não percorremos / Em direção à porta que jamais abrimos / Para o roseiral." [Hacen eco los pasos en la memoria, a lo largo de las galerías que no recorremos en dirección a la puerta que jamás abrimos]. Traducción de Ivan Junqueira. En los versos que anteceden este extracto de Burnt Norton (el primero de los Cuatro Cuartetos), Eliot subraya que los contrafácticos son mera abstracción en nuestra mente. Sin negar esta afirmación, el espíritu del presente artículo es que, asimismo, hay escenarios concebibles que "podrían haber acontecido" y otros que no.
} 
Un escenario contrafáctico es una situación posible que no se realizó. Pero ¿cómo sabemos que una situación es "posible"? Una manera de estipular eso es partir de la consideración de que el futuro está "abierto", o sea, el futuro contempla diversas posibilidades, y no está perfectamente predeterminado.

Por ejemplo, supóngase que mañana el Íbis S.C. va a jugar una partida de fútbol contra el Náutico. Aún siendo el peor equipo del mundo, hay diferentes posibilidades para el resultado del juego. Supóngase que el juego vaya a terminar con el resultado 3 a 2 para el Náutico. Manaña el resultado ya estará definido, pero podremos enunciar contrafácticamente que "sería posible que el Íbis hubiese empatado el juego", por ejemplo si Mauro Shampoo no hubiese perdido aquel gol en el último minuto.

Así, podemos decir que un escenario posible es una posibilidad futura en algún instante to del pasado. De entre las historias posibles (hasta el presente instante), una es "fáctica" (actual, concreta) y las otras son "contrafácticas". Según esta definición, una historia contrafáctica debe ser definida en relación a un instante de ramificación $t_{\mathrm{o}}$ en el pasado. Conforme el instante considerado, las posibilidades son diferentes.

Considerando el ejemplo que abre este artigo, podemos tomar como fecha de ramificación el año 1700. En aquel año, era una posibilidad futura el escenario de varios países independientes de lengua portuguesa (con una probabilidad de, digamos, 80\%). Pero si tomásemos como fecha inicial el año de 1820, esa posibilidade futura tendría una probabilidad bastante reducida.

\title{
2. Contrafácticos en la historia económica
}

\begin{abstract}
A gargalhada era aterrorizadora porque acontecia no passado e só a imaginação maléfica a trazia para o presente, saudade do que poderia ter sido e não foi. ${ }^{1}$

CLARICE LISPECTOR (1977: 48)
\end{abstract}

\footnotetext{
1 "La carcajada era aterradora porque acontecía en el pasado y sólo la imaginación maléfica la traía al presente, nostalgia de lo que podría haber sido y no fue".
} 
El ejemplo más consagrado de análisis contrafáctico viene del área de la historia económica, en el trabajo de Robert William Fogel (1964) sobre las ferrovías y el crecimiento económico de los Estados Unidos en el siglo XIX. Había una concepción tradicional de que las ferrovías habrían sido indispensables para el progreso norteamericano en el siglo XIX, o sea, que ellas habrían sido causa necesaria para este progreso. Fogel examinó esta tesis, calculando minuciosamente los costos y la eficiencia de otras alternativas, y concluyó que si la tecnología ferroviaria no estuviese disponible en la época, había una alternativa igualmente eficiente que era el transporte por hidrovías. Según sus cálculos, ila renta per cápita de hecho alcanzada en los Estados Unidos con ferrovías el 1 de enero de 1890 habría sido alcanzada sin ferrovías (sino con hidrovías) apenas tres meses después! La opción por hidrovías aprovecharía los ríos y lagos navegables, los canales ya construidos, e incluiría varios canales nuevos. La industrialización acabaría siendo más estimulada en regiones diferentes de las de nuestro mundo actual.

Lo que permite que se hagan cálculos económicos sobre escenarios contrafácticos es la posibilidad de hacer previsiones cuantitativas sobre el futuro. Hoy en día, el gobierno puede abrir una licitación en busca de una alternativa energética, y los diferentes proyectos de ingeniería presentan un escenario posible para el futuro. De la manera que caracterizamos a los escenarios contrafácticos (como "posibilidades futuras en un instante pasado”), con el pasar de los años, los proyectos no concretados pueden ser considerados historias contrafácticas. La evaluación retrospectiva que hacemos hoy de un proyecto escrito en un instante $t_{0}$ del pasado permite también una mejor evaluación del correspondiente escenario contrafáctico que la evaluación que se tenga del escenario futuro en $t_{0}$, ya que hoy conocemos el desarrollo fáctico de la coyuntura mundial desde entonces.

\section{Contrafácticos en la historia de la ciencia}

Si hay algo como un sentido de realidad, debe también haber algo como un sentido de posibilidad. [...] Entonces el sentido de posibilidad puede ser prontamente definido 
como la capacidad de pensar como todo podría igualmente ser, sin atribuir mayor importancia a lo que es que a lo que no es. [...] Se dice que tales posibilitadores viven en una tela más fina, en una tela de niebla, imaginación, fantasía y subjuntivo.

ROBERT MUSIL $(1930, \$ 4)^{1}$

Otra área en la que se pueden postular escenarios contrafácticos es la historia de la ciencia. Por ejemplo, en 1826, el joven ingenieron francés Sadi Carnot calculó cuál es la cantidad de trabajo mecánico necesaria para elevar la temperaturade una cierta cantidad de agua en un grado centígrado. iPero, antes de divulgar su resultado, murió de cólera! Sus papeles fueron cajoneados, y reabiertos solamente medio siglo después. Cuando eso aconteció, su resultado ya había sido obtenido por Julius Mayer (1842) y James Joule (1843), tomando el principio de conservación de la energía (un trabajo anterior de Carnot, sobre la eficiencia de máquinas térmicas, acabaría de hecho siendo bastante influyente). ¿Qué habría sucedido si Carnot no hubiese enfermado de cólera? Es razonable suponer que la ciencia de la Termodinámica habría surgido unos diez años antes, y eso tendría varias consecuencias, inclusive para la expansión de las ferrovías.

La postulación de escenarios contrafácticos en la historia de la ciencia es menos precisa de lo que en la historia económica, pues no tenemos cómo prever el futuro de la ciencia, al contrario del relativo control que se tiene en la economía y en la ingeniería. En el caso de la ciencia, podemos tal vez prever aspectos ligados a la política científica y tecnológica, pero no podemos prever que nuevas descubrimientos serían hechos. Por otro lado, cuando miramos al pasado de manera retrospectiva, tenemos la ventaja de saber hoy de qué descubrimientos, los científicos de entonces, estaban próximos. En otras palabras, hoy conocemos mucho mejor la realidad física y biológica que los científicos de entonces estaban investigando. Es eso lo que permite que hoy podamos postular caminos alternativos (contrafácticos) en la historia de la ciencia, de manera más precisa de lo que en la historia social y política.

Este es un campo aún nuevo y controvertido de la filosofía de la ciencia (ver Radick, 2008). ¿Cuál sería el interés en eventos que no sucedieron? El interés es que la postulación de contrafácticos es equivalente a la postulación de

\footnotetext{
${ }^{1}$ Es así que este filósofo de la ciencia convertido en escritor presenta su "hombre sin atributos".
} 
causas. Si alguien afirma que la causa de la llegada de Juan VI al Brasil fue la invasión napoleónica, implícitamente está afirmando la tesis contrafáctica de que si Napoleón no tuviese la intenciín de invadir Portugal, Juan no habría traío la corte al Brasil. Cuando Fogel afirma que si no hubiese ferrovías en los Estados Unidos del siglo XIX, un progreso equivalente habría sido sustentado por hidrovías, está afirmando que las ferrovías no fueron causa necesaria para el gran desarrollo norteamericano.

En el caso de la historia de la ciencia, el presente autor ha investigado los diferentes caminos históricos posibles para llegar a un descubrimiento científico importante, como el nacimiento de la física cuántica (Pessoa, 2000). Los escenarios contrafácticos esbozados están bastante próximos a la historia fáctica, siendo que lo que se investiga es el atraso o adelantamiento en el surgimiento de "avances", que son unidades de conocimiento científico, como ideas, experimentos, datos, formulación de problemas, instrumentos, etc. De una historia posible para otra (de entre aquellas que estamos investigando), los avances son básicamente los mismos, mudando el orden en que ellos aparecen, o el camino causal en que ellos se desarrollan. Eso se justifica en el caso de la historia de las ciencias naturales, pues es razonable suponer que buena parte de los avances refleja un mundo natural real, objetivo, único, que se mantiene constante a pasarse de una historia posible a otra. Que la molécula de ADN tenga una estructura de doble hélice es un hecho objetivo a ser develado en la gran mayoría de las historias posibles de la biología. (La ciencia también tiene aspectos no objetivos, como las "interpretaciones" de la física cuántica, que son más susceptibles a las influencias culturales.)

En el caso de la historia social y política, las posibilidades son mucho más amplias, y por eso la "historia virtual" es mucho más especulativa que una historia contrafáctica de la ciencia, que está más fuertemente constreñida por la existencia de una realidad que la ciencia busca espejar. La historia social y política no tiene ese "atractor" (la realidad natural) que limita la dispersión de las historias posibles. 


\section{Caminos posibles de la evolución biológica}

Me detuve, como es natural, en la frase: Dejo a los varios porvenires (no a todos) mi jardín de senderos que se bifurcan. Casi en el acto comprendí; el jardín de senderos que se bifurcan era la novela caótica; la frase varios porvenires (no a todos) me sugirió la imagen de una bifurcación en el tiempo, no en el espacio. [...] En todas las ficciones, cada vez que un hombre se enfrenta con diversas alternativas, opta por una y elimina las otras; en la del casi inextricable Ts'ui Pên, opta simultáneamente- por todas.

JORGE LUIS BORGES (1941: 119)

En la biología evolutiva, se puede también pensar en historias posibles. Richard Dawkins (2004: 482-93) examinó la cuestión de cómo sería la evolución biológica en la Tierra si se volviese a algún punto del pasado, cuestión ésta que fue tomada también por Stuart Kauffman y por Stephen Jay Gould (1990, cap. 5). El consenso es que las especies que surgirían serían bien diferentes de las actuales, y la especie humana no aparecería en la Tierra. Se puede especular que si el gran meteoro que cayó en la Tierra hace 65 millones de años atrás, y diezmó a los dinosaurios, no hubiese alcanzado la Tierra, ital vez un descendiente del troodon se hubiese vuelto inteligente y estaría aquí ahora escribiendo sobre filosofía de la ciencia! Tal hipótesis fue explorada por el paleontólogo Dale Russell, y otros científicos, como el geólogo S. Conway Morris, consideran que una especie muy parecida al ser humano tendría buenas chances de evolucionar, dado el fenómeno de la evolución convergente, y dadas las restricciones físico-químicas que existen en la generación de nuevas formas de vida por mutación y recombinación. En la evolución convergente, seres de constituiciones diferentes, como mamíferos, reptiles y peces, desarrollan estructuras semejantes, como las alas para volar o el formato hidrodinámico para nadar en la superfície del agua. Podemos decir que los nichos ambientales sirven como "atractores" para el desarrollo de estructuras biológicas, o de "tipos ecológicos".

Sin embargo, quitando la presión de la selección natural, la evolución convergente y las restricciones físico-químicas en la reproducción, nada constriñe la variación de las especies, de forma que los detalles de la evolución 
biológica variarían mucho de una historia evolutiva posible a otra. Esa gran dispersión de historias posibles recuerda aquella de la historia social y política.

Si no es posible prever como será la evolución biológica en el futuro (al contrario del caso de la historia económica) y ni hay un atractor que restrinja el surgimiento de individuos diferentes y que favorezca el mirar retrospectivo (como acontece cuando miramos a la historia pasada de la ciencia con los conocimientos científicops de hoy), ¿qué permitiría especular sobre los caminos posibles de la evolución biológica? En primer lugar, la evolución convergente, que sirve como un atractor general (menos específico que en el caso de la historia de la ciencia); y, en segundo lugar, el conocimiento de la genética y del desarrollo de los individuos biológicos, que permite prever las variaciones que pueden ocurrir en la próxima generación. Combinando ese cálculo, de generación en generación, se podría en principio mapear las posibilidades de evolución biológica, de manera semejante a lo que se puede hacer cuando se mapean las posibles partidas de ajedrez (donde por cada jugada hay un número limitado de lances posibles).

\title{
5. Individuos transmundiales
}

\author{
Se em certa altura \\ Tivesse voltado para a esquerda em vez de para a direita; [...] \\ Seria outro hoje, e talvez o universo inteiro \\ Seria insensivelmente levado a ser outro também. [...] \\ Pode ser que para outro mundo eu possa levar o que sonhei, \\ Mas poderei eu levar para outro mundo o que me esqueci de sonhar? \\ Esses sim, os sonhos por haver, é que são o cadáver. [...] \\ E lá fora o luar, como a esperança que não tenho, é invisível p’ra \\ mim $^{1}$. \\ FERNANDO PESSOA (1928)
}

¿El prefecto de la ciudad de Campinas, Toninho da Costa Santos, podría no haber sido asesinado en septiembre de 2001? Es razonable suponer que sí. Campinas probablemente tendría menos corrupción, la ciudad estaría diferente, y eso podría afectar las circunstancias de la vida de un campinero, como Dogrão.

\footnotetext{
1 "Si a cierta altura hubiese vuelto hacia la izquierda en vez de hacia la derecha; [...] / Sería otro hoy, y tal vez el universo entero / sería insensiblemente llevado a ser otro también. [...] / Puede ser que a otro mundo yo pueda llevar lo que soñé, pero ¿podría llevar a otro mundo lo que me olividé de soñar? / Esos sí, los sueños por tener, es que son el cadáver. [...] / Es allá afuera el lugar, como la esperanza que no tengo, es invisible para mí".
} 
¿En este escenario contrafáctico, Dogrão sería el mismo individuo? ¿Mantendría su identidad?

Esta cuestión es bastante discutida en el área de la filosofia conocida como "metafísica de los mundos posibles" (Loux, 2002, cap. 5). David Lewis, por ejemplo, considera que la contrapartida de Dogrão en un mundo contrafáctico sería un individuo distinto del Dogrão del mundo actual, ya que la contrapartida posee propiedades diferentes de las del Dogrão actual. Otros están en desacuerdo, como Alvin Plantinga, que defiende que Dogrão sería un "individuo transmundial" (el mismo individuo, habitando mundos posibles diferentes), pues a pesar de que sus contrapartidas tienen propiedades accidentales diferentes, todos conservan una misma esencia.

Consideremos la manera por la cual definimos mundos posibles en la sección 1: ¿Cuál sería la solución para esta cuestón de los individuos transmundiales? Según nuestra definición, un escenario posible (que incluye el mundo actual y mundos contrafácticos) sería "una posibilidad futura en algún instante $t_{0}$ del pasado”. Dogrão ayer ponderaba si asistiría al juego de Ponte Preta hoy. Si él estuviera asistiendo, continuará siendo el mismo individuo de ayer, a pesar de que algunas propiedades suyas se hayan modificado; si él hubiera preferido ir al cine, asistir a Efecto Mariposa, ${ }^{1}$ saldrá de la sala de cine en un estado bien diferente del estado de ayer, pero continuará teniendo la misma identidad (si preferimos, podemos convenir que ésta sea la definición de “identidad personal”). O sea, en los dos escenarios posibles, tenemos el mismo individuo Dogrão.

En otros términos, cualquiera que sea la fecha de ramificación $t_{\mathrm{o}}$ en la vida de Dogrão que tomemos para definir mundos posibles, en los diferentes escenarios posibles tendremos el mismo individuo (en situaciones diferentes). Ahora otra cuestión: ¿Y si tomamos una fecha de ramificación anterior a la fecha de nacimiento de Dogrão, o mejor, anterior a la fecha de su concepción? ¡Podremos formular la "paradoja del último trago de la noche2"!

Tomemos $t_{\mathrm{o}}$ como siendo media hora antes del instante de concepción del Dogrão actual. Sus padres estaban bebiendo una cerveza a la noche en la

1 The Butterfly Effect (2004), filme norteamericano escrito y dirigido por E. Bress y J.M. Gruber, que trata de escenarios contrafácticos. Otro filme que involucra esta temática es el francés Smoking/No Smoking (1993), dirigido por Alain Resnais y escrito por A. Jaoui y J.-P. Bacri.

${ }^{2}$ La palabra traducida con la expresión "el último trago de la noche" es saideira (N. del T.). 
playa, decidiendo si salían para el hotel o no. En el mundo actual volverán al hotel, pero hay un escenario plausible en el que habrían decidido tomar una última cerveza, la última de la noche. iEn este mundo posible, habrían llegado una hora más tarde al hotel, y el espermatozoide que acabaría fecundando el óvulo de su madre sería otro! El huevo resultante tendría un código genético diferente del huevo del Dogrão de nuestro mundo actual. Podemos suponer que nacería en la misma fecha, y que también sería llamado Douglas. Cuestón: ¿Habría conservación de la identidad personal? ¿Podríamos decir que se trata del mismo individuo, apenas con propiedades genéticas diferentes? ${ }^{\text {Tा }}$

\section{Agradecimentos}

Este trabajo sería diferente sin las sugerencias de Alexander Pilis, Ana Bia Jesus, Carmem Toledo, Eduardo Kickhöfel, Flávio Tonnetti, Leandro Karnal, Luís Carlos de Menezes, y todo el equipo de Arquitetura Paralaxe: Desaparecer-Aparecer, de la Bienal de São Paulo de 2008, donde este trabajo fue presentado.

\section{Bibliografía}

BORGES, Jorge L. (1941). El jardín de senderos que si bifurcan. In: El jardín de senderos que si bifurcan. Buenos Aires: Ediciones Sur. Republicado em Ficciones (1956).

CAVALCANTE, Rodrigo (2002). E se... a corte portuguesa não tivesse vindo ao Brasil? Superinteressante 173: 32-33.

DAWKINS, Richard (2004). The ancestor's tale. London: Weidenfeld \& Nicolson.

ELIOT, Thomas S. (1935). Burnt Norton. In: Collected poems 1909-1935. London: Faber \& Faber.

FERGUSON, Niall (org.) (1997). Virtual history: alternatives and counterfactuals. London: Macmillan. 
FOGEL, Robert W. (1964). Railroads and American economic growth: essays in econometric history. Baltimore: Johns Hopkins Press.

GOULD, Stephen J. (1990). Vida maravilhosa. Trad. P.C. de Oliveira. São Paulo: Cia. das Letras, São Paulo, cap. 5.

LISPECTOR, Clarice (1977). A hora da estrela. Rio de Janeiro: Rocco.

LOUX, Michael J. (2002). Metaphysics: a contemporary introduction. London: Routledge.

MUSIL, Robert (1930). Mann ohne Eigenschaften. Berlin: E. Rowohlt.

PESSOA, Fernando (1928). Na noite terrível. In: Poesias de Álvaro de Campos. Lisboa: Ática, 1944.

PESSOA Jr., Osvaldo (2000). Histórias contrafactuais: o surgimento da física quântica. Estudos Avançados 14 (39): 175-204.

RADICK, Gregory (2008). Introduction: why what if? Isis 99: 547-51.

RENOUVIER, Charles (1876). Uchronie: l'utopie dans l'histoire. Paris: Bureau de la Critique Philosophique.

ROBERTS, Andrew (org). (2004). What might have been: leading historians on twelve 'what ifs' of history. London: Weidenfeld \& Nicolson.

ROESE, Neal J. (2005). If only. New York: Broadway Books.

ROESE, Neal J. \& OLSON, James M. (orgs.) (1995). What might have been: the social psychology of counterfactual thinking. Mahwah (NJ): Erlbaum.

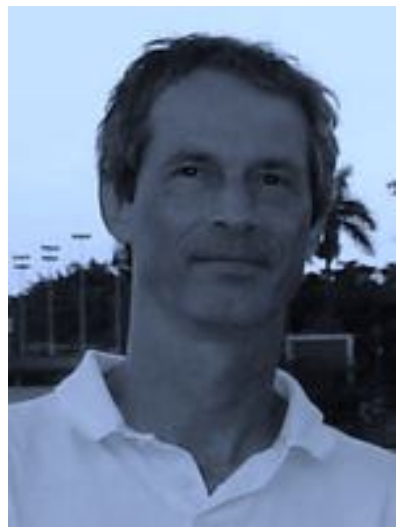

Osvaldo Pessoa Jr. Se formó en Física (1982) y en Filosofía (1984) por la Universidad de São Paulo, hizo la maestría en física experimental en la Universidad Estatal de Campinas (1985), y el doctorado en filosofia de la ciencia en Indiana University (1990), con una tesis sobre la filosofía de la física cuántica. Trabajó en el Posgrado en Enseñanza, Filosofia e Historia de las Ciencias de la Universidad Federal de Bahía y la Universidad Estatal de Feira de Santana. Trabaja desde 2003 en el Departamento de Filosofía, FFLCH, USP, en el área de filosofia de la ciencia. E-mail: opessoa@usp.br. 